\title{
Dip Pen Nanolithography of Conductive Silver Traces
}

\author{
Sheng-Chun Hung, ${ }^{\dagger}$ Omkar A. Nafday, ${ }^{\dagger}$ Jason R. Haaheim, ${ }^{*},{ }^{\dagger}$ Fan Ren,,${ }^{\ddagger}$ G. C. Chi, ${ }^{\S}$ and \\ Stephen J. Pearton ${ }^{\perp}$ \\ NanoInk, 8025 Lamon Avenue, Skokie, Illinois 60077, Department of Chemical Engineering, University of \\ Florida, Gainesville, Florida 32611, Department of Photonics, National Chiao Tung University, \\ Shinchu 302, Taiwan, and Department of Material Science and Engineering, University of Florida, \\ Gainesville, Florida 32611
}

Received: February 18, 2010; Revised Manuscript Received: April 19, 2010

\begin{abstract}
We report the first demonstration of sub $\mu \mathrm{m}$, sub-50- $\mu \Omega \cdot \mathrm{cm}$ conductive traces directly written by Dip Pen Nanolithography (DPN). We achieved sub $\mu \mathrm{m}$ Ag lines with $28.8 \mu \Omega \cdot \mathrm{cm}$ average resistivity after directwrite printing from a silver nanoparticle-based ink suspension and annealing at $150{ }^{\circ} \mathrm{C}$ for $10 \mathrm{~min}$. This compares to Ag bulk resistivity of $1.63 \mu \Omega \cdot \mathrm{cm}$, where the difference is within the range of previously reported variations in conductivity of Ag-based inks due to annealing conditions and larger width scales. We leveraged DPN's ability to directly place materials at specific locations in order to fabricate and characterize these conductive silver $(\mathrm{Ag})$ traces on electrode patterns and multiple substrates $\left(\mathrm{SiO}_{2}, \mathrm{Kapton}\right.$, mica). The low viscosity of the AgNP ink solution allowed write speeds up to $1600 \mu \mathrm{m} / \mathrm{s}$, almost 4 orders of magnitude higher than typical thiol-on-gold DPN writing speeds. This direct-write methodology paves the way for sitespecific deposition of metallic materials for use in applications such as circuit repair, sensor element functionalization, failure analysis, gas sensing, and printable electronics.
\end{abstract}

\section{Introduction}

Metallic nanoparticle (NP) inks offer a versatile, low-cost option to create conductive traces between two electrodes. This ability to "nano-solder" two junctions-or probe disparate elements of pre-existing microcircuitry-lends itself to applications in printed circuit fabrication and flexible electronics, failure analysis of complex microcircuitry, gas sensing, and solar-cell metallization. Common NP based inks consist of solutions of silver $(\mathrm{Ag})$, gold $(\mathrm{Au})$, or copper $(\mathrm{Cu})$, which can be cured at relatively low temperatures $\left(\sim 100-300{ }^{\circ} \mathrm{C}\right)$, and which exhibit low resistivity $(<50 \mu \Omega \cdot \mathrm{cm})$ after deposition and curing. This simple two-step metallization process is especially suitable for low-cost electronics fabrication. However, in the general realm of conductive trace fabrication it is challenging both to achieve precise direct-deposition at specific user-defined sites and to reliably control the dimensions of these metal traces in the $0.5-50.0 \mu \mathrm{m}$ range. Many different approaches already exist to create microscale conductive traces, including drop-ondemand (DOD) inkjet printing, reduction of metal ions in solution, vapor reduction, screen-printing, and microcontact printing $(\mu-\mathrm{CP})$. However, these techniques all have some drawbacks. For example, DOD inkjet printing suffers from ink clogs that form in the nozzle, the minimum feature width $(30-60 \mu \mathrm{m})$ is limited by the minimum nozzle diameter $(1-10$ $\mu \mathrm{m}$ ), and the ink rheology is subsequently constrained by the nozzle dimensions. ${ }^{1}$ Similarly, the resolution of $\mu$-CP is constrained by the limitations of optical lithography, and defects are frequently observed due to issues of stamp/substrate gap and printing force. ${ }^{2}$ More closely related to nanopatterning,

\footnotetext{
* To whom correspondence should be addressed. E-mail: jhaaheim@ nanoink.net. Phone: 847-745-3620. Fax: 847-679-8767.

NanoInk.

* Department of Chemical Engineering, University of Florida.

$\S$ Department of Photonics, National Chiao Tung University.

${ }^{\perp}$ Department of Material Science and Engineering, University of Florida.
}

Wang et al. demonstrated highly controlled deposition of gold nanoparticle (AuNP) sub $\mu$ m lines, but their AuNP traces were neither continuous nor conductive. ${ }^{3} \mathrm{Su}$ et al. demonstrated conductive patterns, but these were with polymers and sol gels which are still quite resistive relative to the high conductivity desired in metallic traces. ${ }^{4,5}$ To truly explore nanoelectronic phenomena, other deposition methods need to be implemented which overcome these drawbacks. Additionally, desirable sitespecific conductive trace fabrication methods should avoid extensive modification of the surface so as to comply with existing electronic fabrication methods.

Dip Pen Nanolithography (DPN) ${ }^{6}$ is a promising candidate for achieving these objectives. A schematic representation of our approach is shown in Figure 1. DPN has been shown to pattern a wide variety of inks on a wide variety of substrates, and thorough reviews of DPN exist in recent literature., Because of its basis as a scanning probe technique, DPN has the unique ability to direct-write traces and register them to existing surface features with nanoscale precision., ${ }^{9,10}$ This capability differentiates DPN as an ideal approach for sub $\mu \mathrm{m}$ decoration of existing microstructures, site-specific device element functionalization, or cosmetic electrical repair of microelectronic elements. Furthermore, DPN is low cost, operates in ambient environment, and does not require physical or chemical modifications of the pre-existing substrate. We herein demonstrate sub $\mu \mathrm{m}$, sub- $50-\mu \Omega \cdot \mathrm{cm}$ DPN-generated conductive traces. We extend our previous work ${ }^{11}$ to show statistically robust line profile control down to $500 \mathrm{~nm}$, with traces that form ohmic contacts with excellent electrical conductivity $(28.80 \mu \Omega \cdot \mathrm{cm}$ average resistivity as measured across 11 separate samples). We chose silver as the NP-based ink for a number of qualities: low bulk resistivity $(\sim 1.6$ $\mu \Omega \cdot \mathrm{cm}$ ), its proven applications as a plasmonic material, ${ }^{12-14}$ and material acceptance in semiconductor fabrication facilities (as opposed to gold, which can contaminate many processes). 


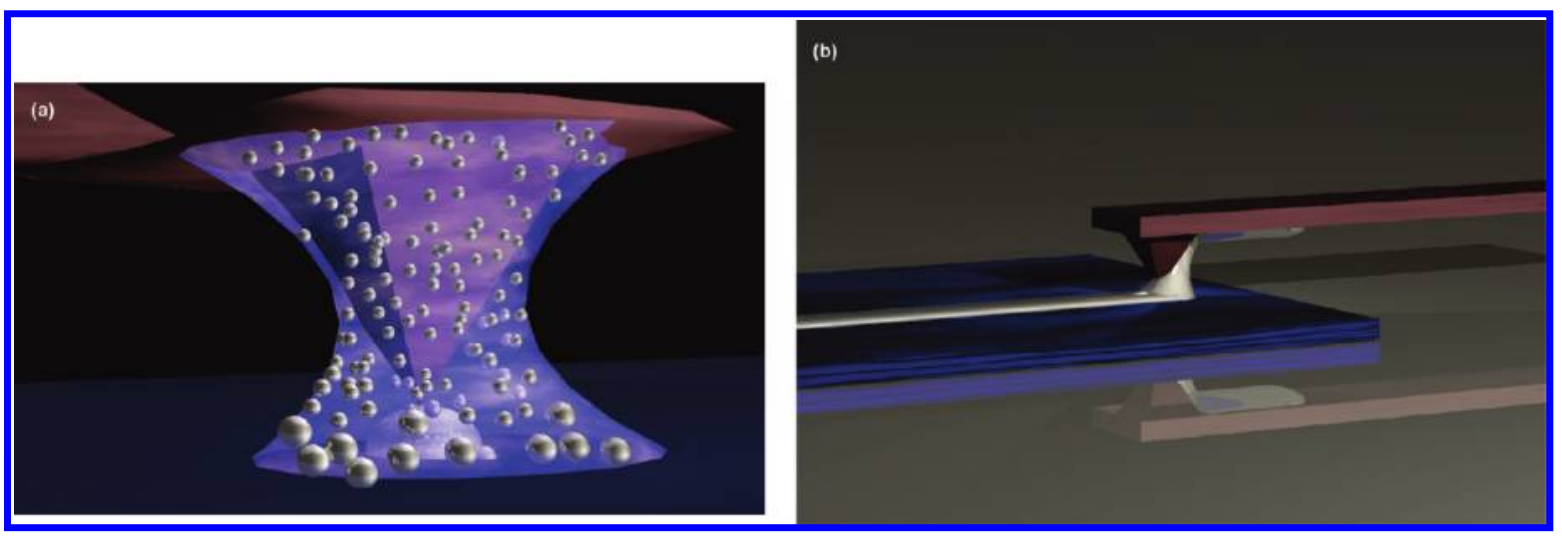

Figure 1. Schematic representation of directly depositing AgNP ink solution onto a generic substrate via DPN: (a) zoomed in perspective showing the necessarily high concentration of silver nanoparticles flowing via a viscous paste meniscus that envelops the tip and (b) zoomed out perspective showing the creation of a continuous silver trace by moving the tip across the surface.

Additionally, a number of fundamental studies have specifically characterized the conductivities of various AgNP inks, investigated the effect of thermal curing on their electrical performance, quantified the effect of surfactant addition on the morphology of the AgNPs, and shown reversible size-tuning of AgNPs. ${ }^{15-19}$ The DPN approach for creating sub $\mu$ m metallic traces is attractive since the process is direct-write and sitespecific, enables versatile pattern creation, is not substratespecific, and does not require harsh operating conditions that would affect pre-existing electronic elements.

\section{Experimental Methods}

Substrates. Our outlook when initiating this work was to write conductive traces with minimum modification of the surface in order to reflect the real-world application of directwriting onto specific features of a chemically/physically sensitive microelectronic device. We used in-house-produced gold electrodes patterned on $\mathrm{SiO}_{2}$ (C-AFM substrates, NanoInk, Skokie, IL), plain silicon, Kapton, and mica substrates throughout this study. We measured the $\mathrm{Au}$-on- $\mathrm{SiO}_{2}$ electrode step height as $\sim 40 \mathrm{~nm}$ (see the Supporting Information, Figure 2) via tapping mode atomic force microscopy (TM-AFM, DPN5000, NanoInk, Skokie, IL). We cleaned the C-AFM substrates by sonicating in acetone and isopropyl alcohol for $5 \mathrm{~min}$ each. We subsequently rinsed the substrates with deionized water and dried with $\mathrm{N}_{2}$. Prior to patterning, we oxygen plasma cleaned the C-AFM substrates for $3 \mathrm{~min}$ to remove organic contamination. For Si-only substrates, we also used RCA (SC1) cleaning; these were used in the process of testing and validating AgNP inks. We rinsed the Kapton substrates with deionized water in order to remove surface contamination, and then dried them with $\mathrm{N}_{2}$. We freshly cleaved mica substrates prior to DPN patterning.

Inks. We used a commercially available hydrophilic AgNP ink (TEC-PA-010, InkTec, South Korea) as the AgNP source in this study (manufacturer specified AgNP size range $<40 \mathrm{~nm}$; AgNP content 55\%; viscosity 7000-7500 cps). Among other reasons, we chose this ink because of its high AgNP concentration. To ensure uniform suspension, we vortexed the AgNP solution for $30 \mathrm{~min}$ prior to patterning in order to avoid phase separation. Since tuning ink viscosity has been shown to play a crucial role in the DPN process of various inks, ${ }^{20,21}$ we performed a "pre-bake" on the ink solution prior to patterning in order to modify its viscosity and make it suitable for DPN printing. This began by shaking the as-received ink container for several seconds in order to mix the ink evenly, and then withdrawing $\sim 5 \mathrm{~mL}$ of ink via pipet. We then gently pipetdeposited the ink onto a sacrificial C-AFM substrate, after which we placed the substrate on a hot plate for $7 \mathrm{~min}$ at $60{ }^{\circ} \mathrm{C}$.

DPN Patterning: Probes and Instrumentation. We oxygen plasma cleaned the silicon nitride $\left(\mathrm{Si}_{3} \mathrm{~N}_{4}\right)$ probes (NanoInk, types $\mathrm{A}, \mathrm{E}$, and $\mathrm{F}$ ) for $20 \mathrm{~s}$ in order to remove organic contamination prior to inking. We then coated the tips with the AgNP ink by directly dipping into a micropipet-deposited droplet on an $\mathrm{SiO}_{2}$ surface, coordinated via the $x-y-z$ stage motors of the patterning tools (NSCRIPTOR and DPN5000 systems, NanoInk, Skokie, IL). We observed an indicative color change on the cantilever as soon as it contacted the ink solution. These same motors then moved the tip to approach the patterning substrate. The patterning process begins with a precisely calibrated initial touch down: we removed excess AgNP ink by creating a "bleeding dot" on first contact and then immediately retracting using the Z-piezo. Carefully monitoring these bleeding dots is essential to writing AgNP patterns of uniform line widths. After initial touch down, and depending on the amount of ink loading on the tip/cantilever, the bleeding dot from a given tip will approach and then maintain a consistent size. At that point in our experiments we initiated line patterning on the given substrate, and/or across the given electrodes. We performed the patterning at ambient conditions ( $\mathrm{RH} 45 \%, 24{ }^{\circ} \mathrm{C}$ ). Force feedback was not necessary for several reasons: this type of physisorbed DPN patterning is essentially force-independent, and we enabled the large-range stage motors to move the sample under the tip for creating lines longer than the $90 \mu \mathrm{m}$ limit of the piezo scanner. Following DPN patterning, we baked the substrate at $150{ }^{\circ} \mathrm{C}$ on a hot plate for $10 \mathrm{~min}$ to cure the AgNP solution and remove any excess solvent. The effect of the curing step on AgNP inks has been well documented in the literature, ${ }^{16}$ wherein resistivity decreases with increasing cure time and temperature. We evaluated the lateral dimensions and topography of the resulting Ag traces via both TM-AFM and scanning electron microscopy (SEM, Hitachi S4800). The postcuring traces were robust enough to withstand intense blasts of dry nitrogen and all subsequent characterization steps.

Electrical Measurements. To fabricate patterns amenable to electrical characterization, we DPN-patterned continuous AgNP traces across at least four gold electrodes on the C-AFM substrate. To circumvent the contact resistance between the probe and electrode, we measured the current-voltage characteristics of the traces with a 4-point probe technique ${ }^{22}$ using a system comprised of a Keithley 2400 sourcemeter, an optical 


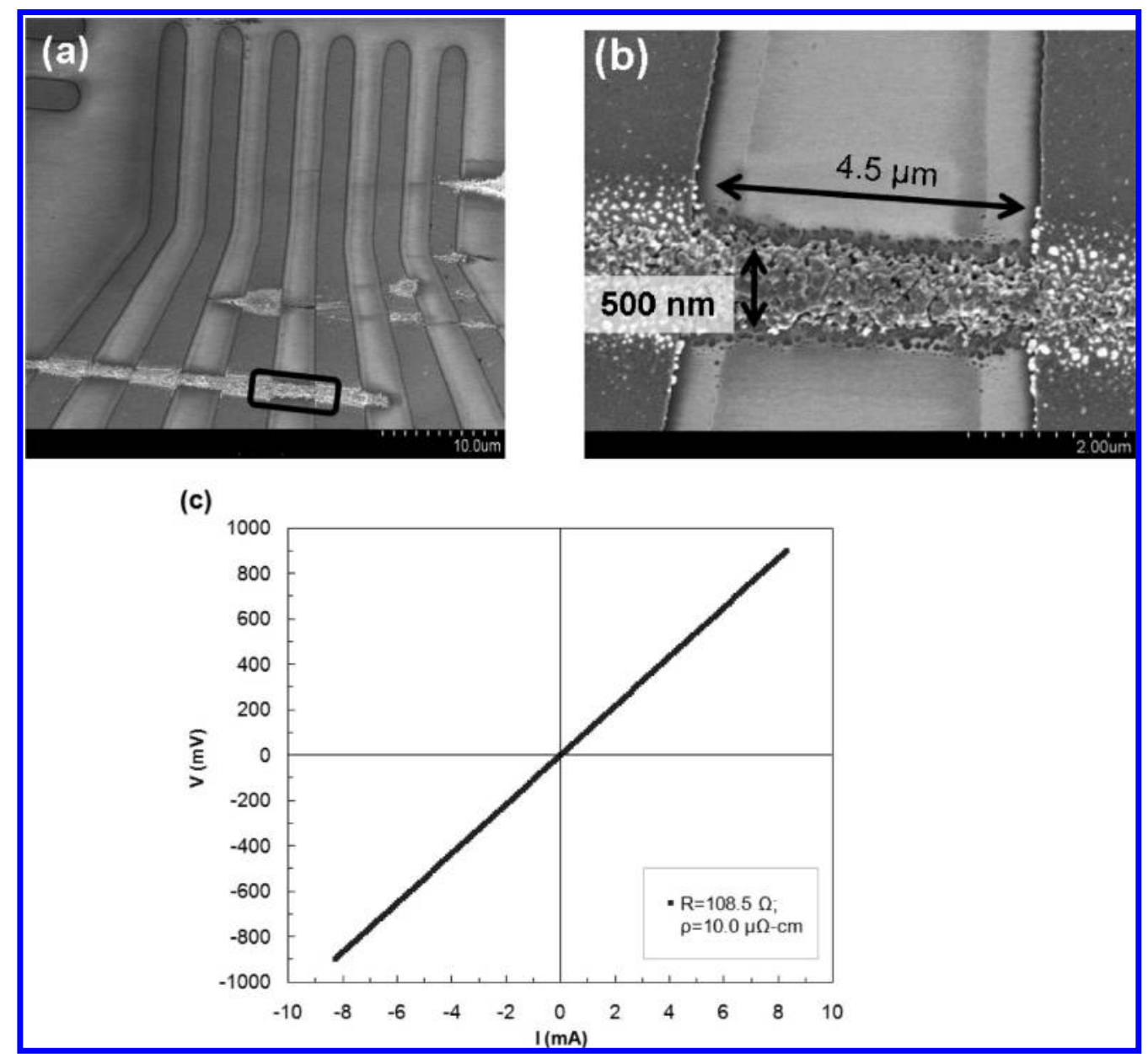

Figure 2. (a) SEM image of a DPN-patterned conductive trace across four electrodes, with several sub $\mu$ m line width interelectrode traces shown. (b) The inset black zoom-box indicated in panel a, where the $500 \mathrm{~nm}$ wide continuous trace spans the $4.5 \mu$ m wide gap between electrodes. (c) $I-V$ curve data- where several hundred data points appear as a continuous line-verifies trace conductivity and yields a trace resistance $R=108.5 \Omega$, with a corresponding resistivity $\rho=10.0 \mu \Omega \cdot \mathrm{cm}$.

microscope, and four micropositioner-mounted needle probes. Typically, when the thickness $t_{\mathrm{w}}$ of a silicon wafer is much less than the diameter of the wafer $d_{\mathrm{w}}$ (i.e., $t_{\mathrm{w}} \ll d_{\mathrm{w}}$ ), we can calculate the sheet resistivity according to:

$$
\rho_{\text {sheet }}=\frac{V}{I} \cdot t_{\mathrm{w}} \cdot \mathrm{CF}(\Omega \cdot \mathrm{cm})
$$

where the correction factor (CF) equals $\pi / \ln (2)$ if the distance between the probes $d_{\mathrm{p}}$ is much less than the diameter of the wafer $d_{\mathrm{w}}$ (i.e., $d_{\mathrm{p}} \ll d_{\mathrm{w}}$ ). For our purposes, we then calculate the resistivity of the AgNP trace according to:

$$
\rho_{\text {trace }}=\frac{R \cdot A}{l}=\frac{V}{I} \cdot \frac{A}{l}=\frac{V \cdot h \cdot w}{I \cdot l}(\Omega \cdot \mathrm{cm})
$$

where $h$ and $w$ are the respective topographic height and line width of the AgNP trace (as measured by TM-AFM). We measured the average AgNP trace height $h$ to be $\sim 200 \mathrm{~nm}$ (Figure 3c), with an example $500 \mathrm{~nm}$ line width $w$ across an electrode shown in Figure 2b. We measured the trace lengths (l) via SEM, and simultaneously corroborated the measured line widths $(w)$ (see the Supporting Information). On the basis of these parameters, we obtained $I-V$ curve data for 11 individual AgNP traces.

\section{Results and Discussion}

We created conductive AgNP traces by moving inked silicon nitride $(\mathrm{SiN})$ probes across various substrates at a specific tip speed. (A schematic depiction is shown in Figure 1b.) Due to the viscosity of this AgNP ink solution, we characterized a tip speed "sweet spot" range in which ink transport to the surface proceeded optimally; we should note that this range $-1400-1600$ $\mu \mathrm{m} / \mathrm{s}$ - is nearly 4 orders of magnitude higher than typical thiolon-gold DPN tip speeds $(0.5-5.0 \mu \mathrm{m} / \mathrm{s}),{ }^{9}$ lending further credence to this ink system's versatility and applicability as a rapid prototyping technique. The kinetics of ink flow from the inked AgNP tip to the substrate-depicted in Figure 1a-are controlled by surface tension and ink viscosity. AgNP ink organization and orientation on the surface, however, is a strictly physisorption-driven phenomena as the AgNP do not experience any affinity toward the surface due to the solvent suspending the nanoparticles. This is a benefit of this ink system as it makes it more widely applicable to a variety of substrates; in this work, we also achieved continuous traces on Kapton tape and mica. While we have characterized many aspects of physisorbed AgNP ink patterning behavior in our previous work $^{11}$ (including diffusive behavior, ink/surface contact angle, and minimum feature size), we herein note that because this AgNP ink is already in the liquid phase, the transport process does not seem to require a water meniscus, and experimental parameters of temperature and relative humidity had virtually no effect on 


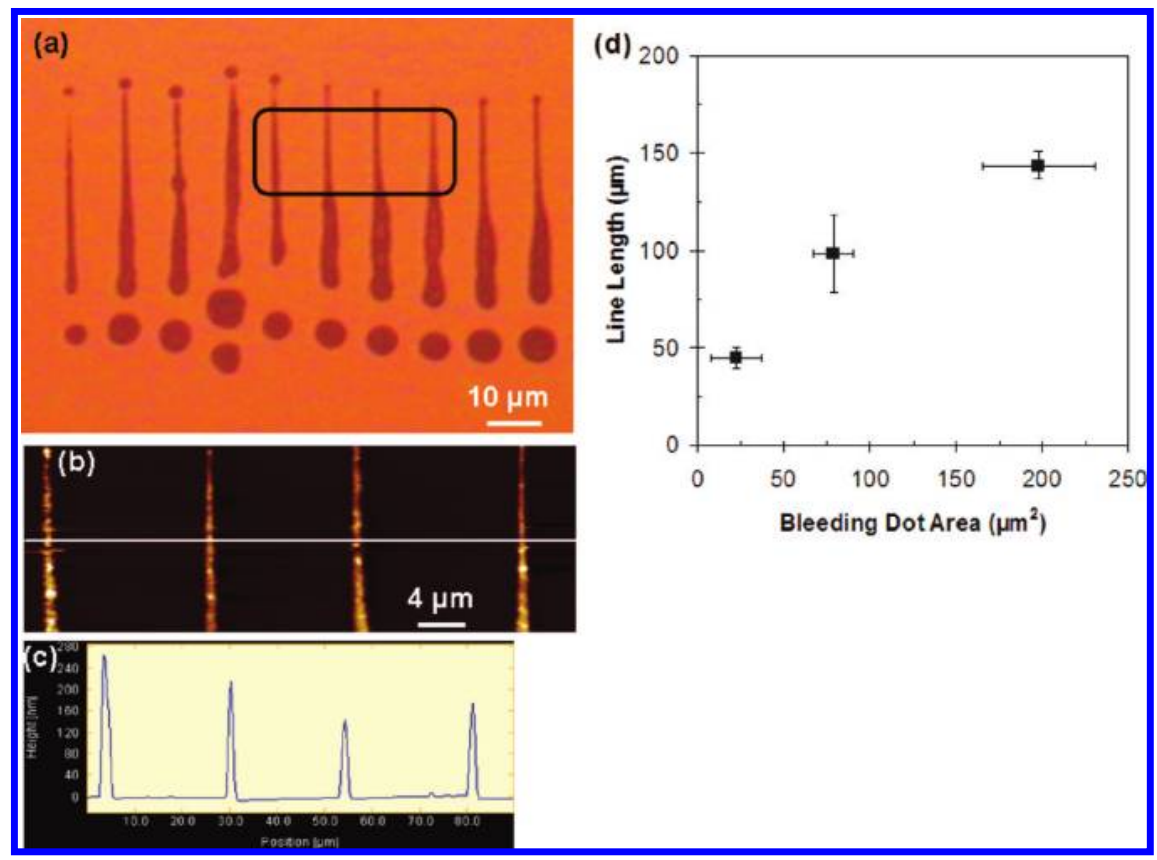

Figure 3. Line patterning results across ten separate experiments. (a) Optical microscopy image of consistent line profiles generated on the same substrate $\mathrm{SiO}_{2}$ from 10 separately inked SiN tips. (b) TM-AFM height image of the lines shown in the inset box and (c) its corresponding line trace profile showing line heights from 120 to $260 \mathrm{~nm}$. (d) Plot examining the roughly linear relationship between the size of the bleeding dot and the resulting line length.

the viscous paste meniscus or the resulting patterns. In the end, proper tuning of ink viscosity, AgNP concentration, and AgNP suspension during DPN are the most important factors leading to continuous and conductive silver traces; suboptimal AgNP concentration or suspension can lead to either discontinuous traces or lack of ink transport from the tip.

Figure 2a shows scanning electron microscopy (SEM) images of conductive silver traces drawn across specific prefabricated electrodes. Figure $2 b$ shows an SEM close-up of a conductive $500 \mathrm{~nm}$ wide silver trace spanning the $4.5 \mu \mathrm{m}$ gap between the gold electrodes. The hydrophilic AgNP ink spreads to broader features on the gold traces due to its lower contact angle on gold, and its overall morphology reflects the high-concentration AgNP clusters fused into continuity via the annealing step. Notably, the trace had no difficulty maintaining continuity up and over the $\sim 40 \mathrm{~nm}$ electrode step height. The $I-V$ behavior of this continuous trace is seen in Figure 2c: the trace is highly conductive, with a resistance $R=108.5 \Omega$ and a corresponding resistivity $\rho=10.0 \mu \Omega \cdot \mathrm{cm}$. Considering that bulk silver $\rho=$ $1.63 \mu \Omega \cdot \mathrm{cm}$, this is an encouraging result: the trace's sub $\mu \mathrm{m}$ scale alone would suggest slightly different electrical performance compared to bulk silver. Furthermore, conductive inks for DOD inkjet printing have widely varying manufactured specifications, and their resulting resistivities have been shown to vary substantially (from $<5$ to $20 \mu \Omega \cdot \mathrm{cm}$ ) based on annealing conditions. ${ }^{12,13,16}$ At the sub $\mu \mathrm{m}$ scale our conductive silver trace performs in the expected range of electrical behavior, but with the benefits of a direct-write methodology.

Nevertheless, our initial outlook was not just to demonstrate the electrical behavior of one sub $\mu \mathrm{m}$ trace but also to validate the robustness of the methodology. To that end, Figure 3a shows highly repeatable deposition and dimensional control of the AgNP ink. We prepared 10 separate $\mathrm{SiN}$ probes, inked nominally identically but at different points in time, and generated 10 continuous adjacent traces on the same $\mathrm{SiO}_{2}$ substrate. The required bleeding dots are shown just below the start of each line trace, drawn from bottom to top. The results demonstrate that by monitoring the initial bleeding dot behavior, it is straightforward to produce continuous traces of consistent line profiles. At our specified tip speed of $1500 \mu \mathrm{m} / \mathrm{s}$, the feature width is controlled by the dynamic depletion of viscous AgNP ink from the tip and cantilever. Writing from thick to thin, the traces become reliably sub $\mu \mathrm{m}$ in their top half (shown in Figure $3 \mathrm{~b}$ ), with overall line lengths consistently greater than $100 \mu \mathrm{m}$. (This is a critical dimension, as microcircuit electrode gaps are often $\sim 40 \mu \mathrm{m}$.) We independently verified that these probes continue writing with similar behavior after being redipped. All of the traces show appreciable height (Figure 3c), and we incorporated these data for subsequent resistivity calculations. Moreover, the data reveal a positive association between initial bleeding dot area and resulting line length (Figure 3d). This supports the intuitive notion that higher ink loading results in a larger initial bleeding dot, and that a higher loaded cantilever will subsequently result in a longer line trace. Thus, by monitoring cantilever loading and the initial bleeding dot size, one can not only ensure a consistent line profile, but also tailor its overall size and length.

With a similar mindset, we set out to demonstrate the electrical reliability of the AgNP DPN methodology. Figure 4a shows $I-V$ curve data generated from 11 separate sets of $\mathrm{SiO}_{2} /$ gold electrodes, covering a range of resistances from $R=0.23$ to $2.10 \Omega$. Deriving the corresponding resistivities as detailed above, we found a range from $\rho=0.8$ to $86.0 \mu \Omega \cdot \mathrm{cm}$ (plotted in Figure $4 \mathrm{~b}$ inset). Further, Figure $4 \mathrm{~b}$ shows the average of $28.80 \pm 8.58 \mu \Omega \cdot \mathrm{cm}$, which compares favorably with the ink manufacturer's specification of $6.0 \mu \Omega \cdot \mathrm{cm}$, and bulk silver $\rho=1.63 \mu \Omega \cdot \mathrm{cm}$. In conjunction with the data from Figure 3, we see a robust method to generate consistent, continuous, and sub-50- $\mu \Omega \cdot \mathrm{cm}$ conductive traces.

Finally, to demonstrate the versatility and applicability of this methodology, we printed continuous traces on both Kapton tape and mica. Panels a and d of Figure 5 show optical images of the traces after curing, with corresponding TM-AFM height images seen in panels $b$ and e of Figure 5. Panels $c$ and $f$ of 


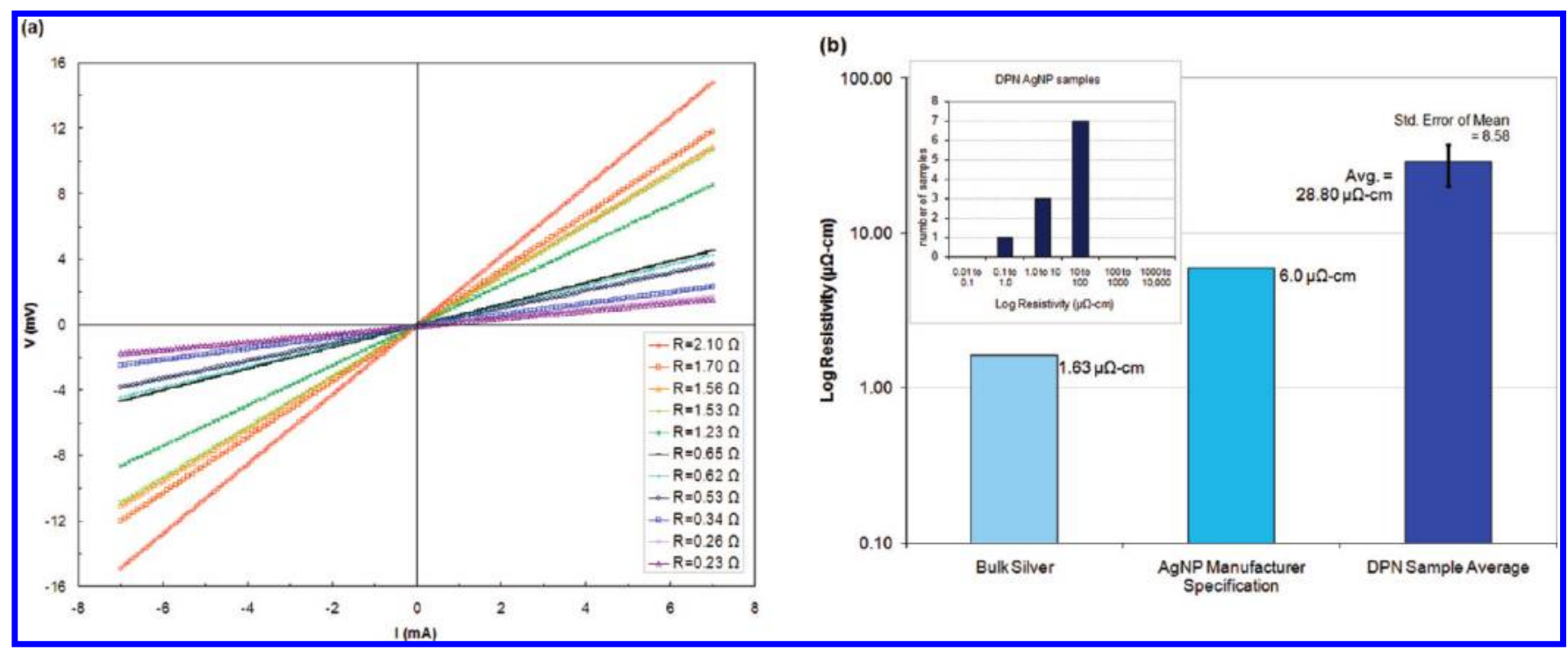

Figure 4. AgNP conductive trace electrical performance data gathered from across 11 separate sets of electrodes: (a) $I-V$ curve data verifies the conductivity of all patterned traces, and shows a range of trace resistance from $R=0.23$ to $2.10 \Omega$. (b) Corresponding resistivity values from the 11 samples are shown in the inset histogram, with an average of $28.80 \mu \Omega \cdot \mathrm{cm}$ and a standard error of the mean of $8.58 \mu \Omega \cdot \mathrm{cm}$. The variation in AgNP cured-trace resistivity is typical of these ink systems ${ }^{16}$ and can be attributed to variations in AgNP morphology, sintering effects, and the curing protocol employed.
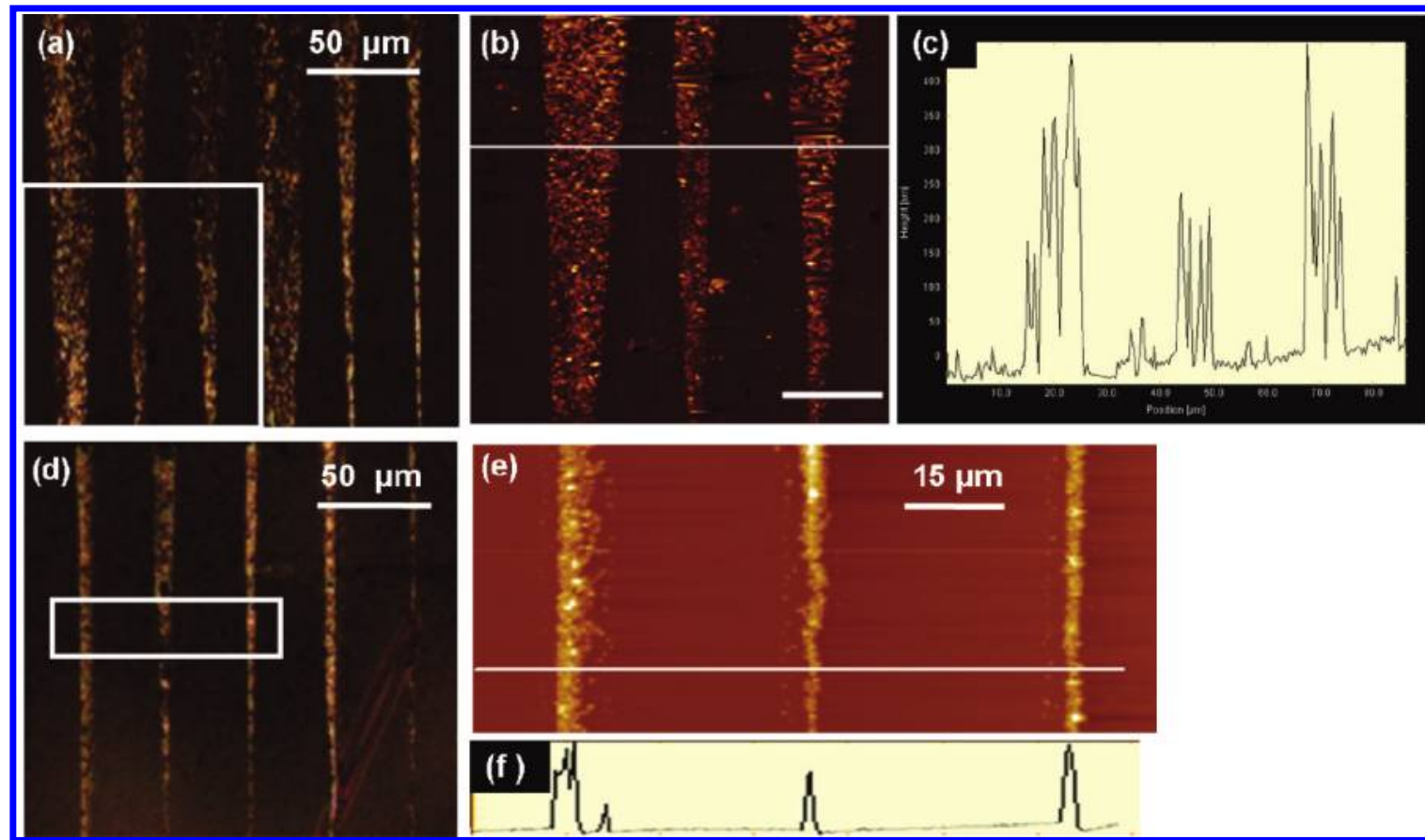

Figure 5. Results that demonstrate the versatility and substrate generality of the DPN conductive trace methodology: (a) optical microscope image of AgNP lines on Kapton tape; (b) TM-AFM height image showing continuous lines of the zoom-box area from panel a; and (c) topographic line trace profiles of panel b. (d) Optical microscope image showing continuous AgNP lines on mica with (e) a TM-AFM image showing the zoom-box area from panel d, and (f) corresponding topographic profiles.

Figure 5 show that, despite being on different substrates, this AgNP ink formed continuous traces with line widths and heights commensurate with the previous $\mathrm{SiO}_{2}$ traces.

\section{Conclusion}

In conclusion, we have demonstrated a reliable method to directly deposit conductive silver traces across specific electrodes using DPN, an approach that is useful for a diverse collection of applications from gas sensing to circuit element failure analysis. DPN provides a new solution both for creating a conductive trace between two specific electrodes and for sub $\mu \mathrm{m}$ decoration of existing microstructures with conductive material. We have provided statistically robust documentation of dimensional pattern control (down to $500 \mathrm{~nm}$ ), and demonstrated electrical performance $(28.80 \mu \Omega \cdot \mathrm{cm}$ average resistivity) that compares favorably with that of bulk silver $(1.63 \mu \Omega \cdot \mathrm{cm})$. We have demonstrated fast writing speeds $(1600 \mu \mathrm{m} / \mathrm{s})$ and described the versatility of this method on additional substrates (Kapton, mica). Future work will include examining the relationship between annealing methods and improved electrical 
performance, pushing the limits of minimum line width, and incorporating these DPN AgNP conductive traces as part of a functioning electronic device.

Acknowledgment. The authors give special thanks to NanoInk colleagues Jae-Won Jang for assistance with 4-point probe measurements and Paul Stiles for assistance with Figure 1 DPN schematic generation.

Supporting Information Available: Full description of the material. This material is available free of charge via the Internet at http://pubs.acs.org.

\section{References and Notes}

(1) Burns, S. E.; Cain, P.; Mills, J.; Wang, J.; Sirringhaus, H. $\underline{M R S}$ Bull. 2003, 28, 829 .

(2) Santhanam, V.; Andrew, R. P. Nano Lett. 2004, 4, 41.

(3) Wang, W. C. M.; Stoltenberg, R. M.; Liu, S. H. ACS Nano 2008, $2,2135$.

(4) Su, M.; Aslam, M.; Fu, L.; Wu, N. Q.; Dravid, V. P. Appl. Phys. Lett. 2004, 84, 21.

(5) Su, M.; Li, S. Y.; Dravid, V. P. J. Am. Chem. Soc. 2003, 125, 33

(6) Piner, R. D.; Zhu, J.; Xu, F.; Hong, S.; Mirkin, C. A. Science 1999 283,5402

(7) Salaita, K. S.; Wang, Y. H.; Mirkin, C. A. Nat. Nanotechnol. 2007, 2,145
(8) Haaheim, J. R.; Nafday, O. A. Scanning 2008, 30, 137.

(9) Haaheim, J. R.; Eby, R.; Nelson, M.; Fragala, J.; Rosner, B.; Zhang, H.; Athas, G. Ultramicroscopy 2005, 103, 117.

(10) Nafday, O. A.; Haaheim, J. R.; Villagran, F. Scanning 2009, 31, 122.

(11) Wang, H. T.; Nafday, O. A.; Haaheim, J. R.; Tevaarwerk, E.; Amro, N. A.; Sanedrin, R. G.; Chang, C. Y.; Ren, F.; Pearton, S. J. Appl. Phys. Lett. 2008, 93, Art. No.143105.

(12) Murray, W. A.; Barnes, W. L. Adv. Mater. 2007, 19, 3771.

(13) Yeshchenko, O. A.; Dmitruk, I. M.; Alexeenko, A. A.; Losytskyy, M. Y.; Kotko, A. V.; Pinchuk, A. O. Phvs. Rev. B 2009, 79, Art. No.235438.

(14) Tai, S. P.; Wu, Y.; Shieh, D. B.; Chen, L. J.; Lin, K. J.; Yu, C. H.; Chu, S. W.; Chang, C. H.; Shi, X. Y.; Wen, Y. C.; Lin, K. H.; Liu, T. M.; Sun, C. K. Adv. Mater. 2007, 19, 4520.

(15) Park, K.; Seo, D.; Lee, J. Colloids Surf., A 2008, 313-314, 351.

(16) Greer, J. R.; Street, R. A. Acta Mater. 2007, 55, 6345.

(17) Park, K. J.; Seo, D. S.; Jang, W. Y.; Lee, J. K. Solid State Phenomena 2007, 124-126, 1205.

(18) Oh, N.; Kim, J. H.; Jin, S.; Yoon, C. H. Small 2009, 5, 1311.

(19) Kalele, S. A.; Kundu, A. A.; Gosavi, G. W.; Deobagkar, D. N.; Deobagkar, D. D.; Kulkarni, S. K. Small 2006, 2, 335.

(20) Giam, L. R.; Wang, Y.; Mirkin, C. A. J. Phys. Chem. A 2009, 3779-3782, 113.

(21) Senesi, A. J.; Rozkiewicz, D. I.; Reinhoudt, D. N.; Mirkin, C. A. ACS Nano 2009, 3,8.

(22) Schuetze, A. P.; Lewis, W.; Brown, C.; Geerts, W. J. American Journal of Physics 2004, 2, 72.

JP101505K 